\title{
GROWTH RATES FOR MONOTONE SUBSEQUENCES ${ }^{1}$
}

\author{
A. DEL JUNCO AND J. MICHAEL STEELE
}

\begin{abstract}
The growth rate of the largest monotone subsequence of a uniformly distributed sequence is obtained. For $a_{n}=n \alpha \bmod 1$ with $\alpha$ algebraic irrational the exponent of growth is found to be precisely the same as for a random sequence.
\end{abstract}

1. Introduction. A well-known result of Erdös and Szekeres [1] states that any sequence of $n$ real numbers contains a monotone subsequence with at least $n^{1 / 2}$ elements. More recently, Hammersley [2] proved that if $l_{n}=$ $l_{n}\left(a_{1}, a_{2}, \ldots, a_{n}\right)$ is the order of the largest increasing subsequence of $a_{1}, a_{2}, \ldots, a_{n}$, and the $a_{i}$ are chosen independently with the uniform distribution on $[0,1]$, then

$$
\lim _{n \rightarrow \infty} n^{-1 / 2} l_{n}=C,
$$

where $C$ denotes a constant and the convergence is in probability. This result was strengthened by Kesten [4] to provide almost sure convergence, and Logan and Shepp [6] proved that $C \geqslant 2$. Our objective here is to provide results like (1) for sequences which are uniformly distributed in $[0,1]$, but which are not random. Of particular interest to us is the sequence $a_{n}=$ $n \alpha \bmod 1$ where $\alpha$ is an algebraic irrational.

2. Uniformly distributed sequences. We will denote by $1_{[a, b)}(x)$ the indicator function of the interval $[a, b)$ and will say a sequence $\left(a_{n}\right)$ is uniformly distributed in $[0,1]$ provided for all $0 \leqslant a<b \leqslant 1$,

$$
\lim _{n \rightarrow \infty} n^{-1} \sum_{i=1}^{n} 1_{[a, b)}\left(a_{i}\right)=b-a \text {. }
$$

The best one can say about the growth rate of $l_{n}$ for a general uniformly distributed sequence is the following:

THEOREM 1. If $\left(a_{n}\right)$ is uniformly distributed, then

$$
\lim _{n \rightarrow \infty} n^{-1} l_{n}=0 \text {. }
$$

Proof. Let $A$ and $n$ be positive integers and for $0 \leqslant i \leqslant A-1$ and

Received by the editors July 25, 1977 and, in revised form, September 16, 1977.

AMS (MOS) subject classifications (1970). Primary 10K05, 10K30.

Key words and phrases. Monotone subsequence, uniform distribution, algebraic irrationals, discrepancy.

${ }^{1}$ Supported by Contract \#67-8473. 


$$
\begin{aligned}
& 0 \leqslant j \leqslant A-1 \text { let } \\
& \quad S_{i j}=\left\{k: 1 \leqslant k \leqslant n, i A^{-1} \leqslant a_{k}<(i+1) A^{-1},\right. \\
& \left.\qquad j n A^{-1}+1 \leqslant k \leqslant(j+1) n A^{-1}\right\} .
\end{aligned}
$$

By $\left|S_{i j}\right|$ we denote the cardinality of $S_{i j}$ and we set $g(n)=\max _{i, j}\left|S_{i j}\right|$. If $n$ tends to infinity along the subsequence $n=\gamma A, \gamma=1,2, \ldots$, then $g(n) / n$ is easily seen to converge to $A^{-2}$ by the uniform distribution of $\left(a_{n}\right)$.

Next let $S=\left\{i_{1}<i_{2}<\cdots<i_{s}\right\}$ be any subsequence of $1,2, \ldots, n$ such that $a_{i_{1}} \leqslant a_{i_{2}} \leqslant \cdots \leqslant a_{i_{s}}$. We note that $S$ intersects at most $2 A-1$ of the $S_{i j}$. (One can identify $a_{1}, a_{2}, \ldots, a_{n}$ with its graph in $\{1,2, \ldots, n\} \times$ $[0,1]$ and view the $S_{i j}$ as "boxes.") This observation yields the inequality $|S| \leqslant 2 A g(n)$, and since $l_{n} \leqslant|S|$ we have $\overline{\lim }_{n \rightarrow \infty} l_{n} / n \leqslant 2 / A$ provided the limit is taken along the subsequence $n=k A$.

For $k A<n<(k+1) A$ we note that

$$
\begin{aligned}
l\left(a_{1}, a_{2}, \ldots, a_{n}\right) & \leqslant l\left(a_{1}, a_{2}, \ldots, a_{A k}\right)+l\left(a_{A k+1}, \ldots, a_{n}\right) \\
& \leqslant l\left(a_{1}, a_{2}, \ldots, a_{A k}\right)+A .
\end{aligned}
$$

This proves

$$
\varlimsup_{n \rightarrow \infty} \frac{l_{n}}{n} \leqslant \varlimsup_{k \rightarrow \infty} \frac{\left(l_{k A}+A\right)}{k A} \leqslant \frac{2}{A},
$$

which completes the proof of (1), since $A$ was an arbitrary positive integer.

3. Results concerning $(n \alpha)$. To show that $l_{n}=o(n)$ is best possible we do not have to go out of the class of sequences $a_{n}=n \alpha \bmod 1$.

THEOREM 2. Let $C_{n}$ be a sequence of real numbers such that $C_{n} \rightarrow 0$ as $n \rightarrow \infty$; then there is a transcendental $\alpha$ such that for $a_{n}=n \alpha \bmod 1$ we have

$$
n^{-1} l_{n} \geqslant C_{n} \text { for infinitely many } n \text {. }
$$

Proof. The proof depends on an elementary lower estimate for $l_{n}$ in terms of the denominators $q_{k}$ of the convergents $p_{k} / q_{k}$ of $\alpha$. First we assume $n=q_{k+1}$ and that $\left\{q_{k} \alpha\right\}>0$, where $\{x\}=x-\left[x+\frac{1}{2}\right]$. For $j=S q_{k}$ the sequence $j \alpha$ with $S=1,2, \ldots,\left[q_{k+1} / q_{k}\right]$ can be viewed as making small positive steps, so we have the lower bound

$$
l_{n} \geqslant \min \left(1 /\left\{q_{k} \alpha\right\}, q_{k+1} / q_{k}\right) .
$$

By the standard theory of continued fractions (e.g., [3, p. 9]) we have $\left|\left\{\alpha q_{k+1}\right\}\right|<1 / q_{k+1}$, so (4) implies $l_{n} \geqslant q_{k+1} / q_{k}$. Since $C_{n} \rightarrow 0$ we can choose $q_{k}$ which go to infinity as rapidly as we like such that $1 / q_{k} \geqslant C_{t}$ for $t=q_{k+1}$. In particular, we may require $q_{k}$ to grow rapidly enough to insure that $\alpha$ is transcendental. Finally, we note that if the condition $\left\{q_{k} \alpha\right\}>0$ is not met by infinitely many $k$, we need only replace $\alpha$ by $1-\alpha$. This will then complete the proof.

There is a more precise result which can be proved if $\alpha$ is algebraic. To state it succinctly, we let $l_{n}^{\prime}$ denote the order of the largest monotone 
(increasing or decreasing) subsequence of $a_{1}, a_{2}, \ldots, a_{n}$.

THEOREM 3. If $a_{n}=n \alpha \bmod 1$ where $\alpha$ is an algebraic irrational, then

$$
\lim \left(\log l_{n}^{\prime}\right) /(\log n)=1 / 2 \text {. }
$$

Proof. We must obtain quantitative versions of the estimates used in Theorem 1. To begin, for $0 \leqslant i \leqslant n-1$ and $0 \leqslant j \leqslant n-1$ we let

$$
S_{i j}=\left\{a_{k}: i / n \leqslant a_{k}<(i+1) / n, j n+1 \leqslant k \leqslant(j+1) n\right\}
$$

and observe that

$$
\max _{i, j}\left|S_{i j}\right| \leqslant \max _{0<j<n-1}\left\{1+2 n D_{n}^{j}\right\}
$$

where

$$
D_{n}^{j}=\sup _{0<x<1}\left|n^{-1} \sum_{k=j n+1}^{(j+1) n} 1_{[0, x)]}\left(a_{k}\right)-x\right| .
$$

Also, if $S=\left\{a_{i}, a_{i_{2}}, \ldots, a_{i_{s}}\right\}$ is any monotone subsequence of $\left\{a_{1}, a_{2}, \ldots, a_{n^{2}}\right\}$, we know $S$ intersects at most $2 n-1$ of the $S_{i j}$. Thus, we have

$$
n \leqslant l_{n^{2}}^{\prime} \leqslant 2 n \max _{i, j}\left|S_{i j}\right|
$$

where the first inequality follows from the Erdös-Szekeres theorem mentioned in the introduction.

Since the sets $\{(j n+1) \alpha,(j n+2) \alpha, \ldots,(j+1) n \alpha\}, j=0,1, \ldots, n-1$, are translates of $\{\alpha, 2 \alpha, \ldots, n \alpha\}$, we have

$$
\max _{0<j<n-1} D_{n}^{j}=O\left(D_{n}^{1}\right) \text {. }
$$

By the Thue-Siegel-Roth theorem [5, pp. 122-124] we know that $D_{n}=D_{n}^{1}=$ $O\left(n^{-1+\varepsilon}\right)$ for all $\varepsilon>0$. This fact, with (7) and (8), yields

$$
\lim _{n \rightarrow \infty}\left(\log l_{n^{2}}^{\prime}\right) /(\log n)=1
$$

For the final step choose $n$ so that $n^{2} \leqslant j<(n+1)^{2}$ and note $l_{n^{2}}^{\prime} \leqslant l_{j}^{\prime} \leqslant l_{n^{2}}$ $+2 n$. By the bounds on $j$ and the limit in (9), one completes the proof with a brief computation.

There are two corollaries of the proof of Theorem 3.

Corollary. 1. If $\alpha$ is an irrational for which $D_{n}=O\left(n^{-1+\varepsilon}\right)$ for all $\varepsilon>0$, then (5) holds. In particular, this is the case if $\alpha$ is of finite type 1.

Corollary 2. For all $\alpha$ except a set of measure 0 , one has (5).

The proof of Corollary 2 depends only on the fact that $D_{n}=O\left(n^{-1+\varepsilon}\right)$ for all $\varepsilon>0$ and almost every $\alpha$. (For more precise results on $D_{n}$, see Niederreiter [7]).

Acknowledgement. We wish to thank Professors $\mathrm{H}$. Kesten and $\mathrm{H}$. Niederreiter for their comments on an earlier draft of this paper. 


\section{REFERENCES}

1. P. Erdös and G. Szekeres, A combinatorial problem in geometry, Compositio Math. 2 (1935), 463-470.

2. J. M. Hammersley, A few seedlings of research, Proc. Sixth Berkeley Sympos. Math. Statist. and Probability, Univ. of California Press, Berkeley, Calif., 1972.

3. A. Ya. Khinchin, Continued fractions, Univ. of Chicago, Chicago, Ill., 1964.

4. H. Kesten, Comment to "Subadditive ergodic theory" by J. F. C. Kingman, Ann. Probability 1 (1973), 903.

5. L. Kuipers and H. Niederreiter, Uniform distribution of sequences, Wiley, Toronto, 1974.

6. B. F. Logan and L. A. Shepp, A variational problem for random Young tableaux, Advances in Math. 26 (1977), 206-222.

7. H. Niederreiter, Metric theorems on the distributions of sequences, Proc. Sympos. Pure Math., vol. 24, Amer. Math. Soc., Providence, R. I., 1973, pp. 195-212.

Department of Mathematics, Ohio State University, Columbus, Oho 43210

Department of Mathematics, Universtty of British Columbia, Vancouver V6T IW5, B. C., Canada 